\title{
CRUSTÁCEOS COMO COMPONENTES PRINCIPALES EN LA DIETA DE PRIONOTUS RUBIO (SCORPAENIFORMES: TRIGLIDAE) EN LAS BARRANCAS, ALVARADO, EN EL ESTADO DE VERACRUZ, MÉXICO
}

\section{Crustaceans as main component of the Prionotus rubio diet (Sorpaeniformes: Triglidae) of Las Barrancas, Alvarado in the State of Veracruz, Mexico}

\author{
Néstor Armando Quijano Reyes ${ }^{1 a^{*}}$, Manuel Ortíz ${ }^{2 a}$, José Antonio Martínez Pérez ${ }^{1 b}$, \\ Jonathan De la Cruz-Torres ${ }^{1 \mathrm{c}}$, Ariana Solís Gómez ${ }^{1 \mathrm{~d}}$, Jairo Sarael Garduño-Gaona ${ }^{1 \mathrm{e}, 2 \mathrm{~b}}$, \\ Jonathan Franco López ${ }^{1 \mathrm{f}}$ y Brenda Guadalupe Martínez-Hernández ${ }^{1 \mathrm{~g}}$
}

${ }^{1}$ Laboratorio de Zoología, edificio L2, Facultad De Estudios Superiores Iztacala, UNAM, C.P. 54090, Av. de los Barrios Número 1, Col. Los Reyes Iztacala, Tlalnepantla, Estado de México, México. ${ }^{1 a}$ (D) orcid.org/00000001-5077-9487; *Para correspondencia: armquire@gmail.com; 1b Dorcid.org/0000-0002-9132-4777; lc (D) orcid.org/0000-0001-7856-7638; ld Dorcid.org/0000-0003-3833-6602; le (D) orcid.org/0000-00024417-3443; if (D) orcid.org/0000-0002-6006-6031; 1g Dorcid.org/0000-0003-3683-5045. 2Laboratorio de crustáceos, edificio L3, Facultad De Estudios Superiores Iztacala, UNAM, C.P. 54090, Av. de los Barrios Número 1, Col. Los Reyes Iztacala, Tlalnepantla, Estado de México, México. ${ }^{2 a}$ (D orcid.org/0000-0002-6985-8019.

\section{RESUMEN}

La alimentación de Prionotus rubio (Actinopterygii: Scorpaeniformes: Triglidae) se estudió sobre los ejemplares obtenidos en aguas del Golfo de México. Se realizaron dos muestreos en los meses de marzo y octubre del 2018, en Las Barrancas, Alvarado, Veracruz; con el uso de un chinchorro playero de $800 \mathrm{~m}$ de largo. Se obtuvo un total de 67 peces de la especie P. rubio; se extrajeron los contenidos estomacales y se procesaron los datos mediante diversos índices. En el primer muestreo se encontró un total de 19 tipos alimentarios, solo uno perteneció al grupo de los peces y otro al de los moluscos, el resto perteneció al subfilo Crustacea. En contraste a la colecta del segundo muestreo, se encontraron al menos tres especies de peces, cinco táxones de moluscos, y el resto fueron de crustáceos, sumando un total de 22 tipos alimentarios y 18 distintos a los del primer muestreo. El índice de Levins arrojó un resultado de 0.69 , lo que considera que esta especie no tiene preferencia sobre algún grupo para alimentarse. De acuerdo a lo observado, se concluye que ésta especie es carnívora y se alimenta principalmente de crustáceos y peces, dependiendo de la temporada.

Palabras clave: alimentación, Triglidae, Prionotus, bentófagos.

\section{ABSTRACT}

The feeding of Prionotus rubio (Actinopterygii: Scorpaeniformes: Triglidae) was studied on specimens obtained in Gulf of Mexico Waters. Two samplings were carried out in the months of march and october of 2018, in Las Barrancas, Alvarado, Veracruz; with the use of an $800 \mathrm{~m}$ long trawl. A total of 67 fish of the species P. rubio was obtained; the stomach contents were extracted and the data were processed by various index. In the first sampling, a total of 19 food items were found, only one belonged to the group of fish and another to the group of mollusks, the rest belonged to the subphylum Crustacea. In contrast to the collection of the second sampling, at least three species of fish were found, 5 mollusks, and the rest were crustaceans, totaling 22 food items and 18 different from those of the first sample. The Levins index yielded a result of 0.69 , which considers that this species does not have preference over any according to the observations, it is concluded that this species is carnivorous and feeds mainly on crustaceans and fishes, depending on the season. 


\section{INTRODUCCIÓN}

La familia Triglidae se caracteriza por tener la región cefálica angulosa, armada con numerosas espinas y con fuertes proyecciones en el hocico (Hoese y Moore, 1977). El nombre de la familia hace referencia a la forma de las aletas pectorales, las cuales presentan los tres primeros radios separados del resto; al moverlos sobre el sustrato, estos radios detectan estímulos mecánicos y químicos, siendo así como encuentran su alimento (Randall, 1983; Silver y Finger, 1984).

Los peces más representativos dentro de la familia Triglidae, en el Golfo de México, son especies del género Prionotus (Miller y Richards, 1977), los cuales se alimentan de diversos camarones, cangrejos, jaibas, y en algunos casos, como Prionotus stearnsi y P. rubio, también incluyen a los peces óseos como una parte importante de su dieta (Lewis y Yerger, 1976; Ross, 1983; Cruz-Escalona et al., 2001). Algunos de estos crustáceos son de importancia comercial, especialmente varias especies de camarón y jaiba, ya que constituyen una fracción indispensable para las pesquerías en México (Peláez-Rodríguez et al., 2005). Debido a que comparten hábitat, los pescadores de camarón y jaiba suelen encontrar entre sus redes organismos del género Prionotus, los cuales se catalogan como fauna de acompañamiento, y suelen ser desechados. Por mencionar algunos ejemplos, en el estado de Campeche, se ha calculado que este tipo de fauna llega a superar hasta 6 veces la del camarón, en peso por captura (Ramírez-Rodríguez, 2015). Otros estudios demuestran que en áreas subtropicales de México, en la pesca de camarones, la proporción extraída es de cinco toneladas métricas de pez por una de camarón, e incluso datos de diferentes localidades en el mundo muestran que en algunos tipos de pesquerías más del $90 \%$ de la pesca total es fauna de acompañamiento (Peláez-Rodriguez et al., 2005).

La fauna de acompañamiento no se considera un recurso pesquero, sin embargo, esto podría replantearse tras estudios de índole ecológico y trófico, que generen las bases para conocer mejor este posible recurso; es así que el objetivo del presente estudio fue analizar la composición de la dieta, su variación entre las temporadas de lluvias y estiaje, así como la importancia de cada tipo alimentario que forma parte de la alimentación de P. rubio en la localidad de Las Barrancas en el Estado de Veracruz, México.

\section{OBJETIVO}

- Analizar la composición de la dieta de Prionotus rubio, su variación en dos tiempos de muestreo y la importancia de cada tipo alimentario, en Las Barrancas, Estado de Veracruz, México.

\section{MATERIALES Y MÉTODOS}

Se realizaron dos muestreos, el primero en marzo y el segundo en octubre del 2018, en Las Barrancas, perteneciente al municipio de Alvarado, en el Estado de Veracruz. El material fue obtenido mediante un chinchorro playero de $800 \mathrm{~m}$ de largo con una abertura de malla de dos pulgadas. Se transportaron con hielo al laboratorio de zoología de la Facultad de Estudios Superiores Iztacala (FESI), donde se pesaron los organismos con una balanza semianalítica con precisión de $0.01 \mathrm{~g}$. Posteriormente se realizó la disección y se almacenó el contenido estomacal en frascos con alcohol al $70 \%$. Los tipos alimentarios fueron determinados hasta el nivel taxonómico más bajo posible empleando bibliografía especializada que se cita a continuación. Para la determinación de crustáceos: Gore y Scotto (1979); Manning (1969); Abele y Kim (1986); Ortiz y Lalana (2010) y Ng et al. (2008). 
Para peces: Richards y Miller (2002) y McEachran y Fechhelm (2005), y finalmente, para los moluscos: Abbott (1974) y Tunnell Jr et al. (2010). Se obtuvo el Índice de Importancia Relativa (IIR) propuesta por Pinkas et al. (1971), para esto se registró: frecuencia, número de organismos y peso húmedo, como lo indica la técnica de Hyslop (1980). El criterio para el número de organismos se basó en la cuantificación de cabezas, en caso de que estas no se encontraran, se contó como a un individuo si se encontraba más del $50 \%$ del organismo. El índice de Levins se calculó con la fórmula propuesta por Krebs (1999), para ambas temporadas, y finalmente, para calcular el nivel trófico se siguió el método propuesto por Cortés (1999).

\section{RESULTADOS Y DISCUSIÓN}

Durante los dos períodos de muestreo se obtuvieron 28 organismos en la primera salida y 39 en la segunda, sumando un total de 67 estómagos de organismos pertenecientes a Prionotus rubio. Solo seis estómagos se encontraron vacíos o con materia orgánica no identificable (MONI).

En cuanto a la composición de la dieta de Prionotus rubio, se encontraron 36 tipos alimentarios en las temporadas de muestreo (Tabla I); todos ellos forman parte del bentos, a excepción de los organismos de la familia Engraulidae. En comparación a los resultados planteados por Cruz-Escalona et al. (2001), que reportaron 22 para la misma especie, a pesar de haber realizado más muestreos, en los meses de septiembre de 1994 a agosto de 1995 con excepción de mayo, junio, diciembre y febrero. Al igual que en este trabajo, dichos tipos alimentarios pertenecieron a los mismos grandes grupos, crustáceos, moluscos y peces, pero sin una variación en la composición de la dieta, a diferencia de lo encontrado en el presente estudio, en el que se observó una predominancia de los crustáceos en el primer muestreo y de peces en el segundo.

Tabla I. Entidades alimentarias y valores que conformaron el índice de importancia relativa, según Pinkas et al. (1971). Los tipos alimentarios están ordenadas por el \% de IIR, de mayor a menor.

\begin{tabular}{|c|c|c|c|c|c|c|c|c|}
\hline Entidad & $\mathbf{N}$ & N \% & $\begin{array}{c}\text { Peso } \\
\text { (g) }\end{array}$ & P \% & FO & FO \% & IIR & IIR \% \\
\hline Anchoa sp. & 15 & 8.67052023 & 18.05 & 26.046176 & 4 & 4.54545455 & 157.803165 & 20.832494 \\
\hline $\begin{array}{l}\text { Dendobranquiados } \\
\text { sin identificar }\end{array}$ & 12 & 6.93641618 & 2.22 & 3.2034632 & 7 & 7.95454545 & 80.6581315 & 10.648139 \\
\hline $\begin{array}{l}\text { Esquílidos } \\
\text { sin identificar }\end{array}$ & 22 & 12.716763 & 2.13 & 3.07359307 & 4 & 4.54545455 & 71.774358 & 9.47533991 \\
\hline Sicyonia typica & 10 & 5.78034682 & 6.08 & 8.77344877 & 4 & 4.54545455 & 66.1536163 & 8.73331542 \\
\hline Squilla sp. & 13 & 7.51445087 & 1.33 & 1.91919192 & 5 & 5.68181818 & 53.6002431 & 7.07607317 \\
\hline Anchelous sp. & 11 & 6.3583815 & 3.52 & 5.07936508 & 4 & 4.54545455 & 51.9897572 & 6.86346376 \\
\hline $\begin{array}{l}\text { Platylambrus } \\
\text { serratus }\end{array}$ & 9 & 5.20231214 & 2.46 & 3.54978355 & 4 & 4.54545455 & 39.7822531 & 5.25188167 \\
\hline $\begin{array}{l}\text { Engraulidos } \\
\text { sin identificar }\end{array}$ & 4 & 2.31213873 & 4.22 & 6.08946609 & 4 & 4.54545455 & 38.1891128 & 5.04156214 \\
\hline
\end{tabular}


Tabla I. Continuación

\begin{tabular}{|c|c|c|c|c|c|c|c|c|}
\hline Entidad & $\mathbf{N}$ & N \% & $\begin{array}{c}\text { Peso } \\
\text { (g) }\end{array}$ & P \% & FO & FO \% & IIR & IIR \% \\
\hline Callinectes danae & 3 & 1.73410405 & 6.97 & 10.0577201 & 2 & 2.27272727 & 26.7996002 & 3.53796776 \\
\hline Penaeus sp. & 6 & 3.46820809 & 2.11 & 3.04473304 & 3 & 3.40909091 & 22.2032084 & 2.93117192 \\
\hline $\begin{array}{l}\text { Braquiros } \\
\text { sin identificar }\end{array}$ & 4 & 2.31213873 & 1.35 & 1.94805195 & 4 & 4.54545455 & 19.3645031 & 2.55641827 \\
\hline $\begin{array}{l}\text { Porichthys } \\
\text { plectrodon }\end{array}$ & 6 & 3.46820809 & 0.33 & 0.47619048 & 4 & 4.54545455 & 17.9290844 & 2.36692048 \\
\hline $\begin{array}{l}\text { Callinectes } \\
\text { bocourti }\end{array}$ & 2 & 1.15606936 & 3.19 & 4.6031746 & 2 & 2.27272727 & 13.0891908 & 1.72797858 \\
\hline $\begin{array}{l}\text { Estomatópodos } \\
\text { sin identificar }\end{array}$ & 4 & 2.31213873 & 0.37 & 0.53391053 & 4 & 4.54545455 & 12.9365876 & 1.70783256 \\
\hline $\begin{array}{l}\text { Achelous } \\
\text { depressifrons }\end{array}$ & 5 & 2.89017341 & 0.39 & 0.56277056 & 3 & 3.40909091 & 11.7713999 & 1.5540095 \\
\hline Achelous ordwayi & 3 & 1.73410405 & 4.06 & 5.85858586 & 1 & 1.13636364 & 8.62805671 & 1.13903888 \\
\hline $\begin{array}{l}\text { Hepatus } \\
\text { pundibundus }\end{array}$ & 9 & 5.20231214 & 1.47 & 2.12121212 & 1 & 1.13636364 & 8.32218666 & 1.09865923 \\
\hline $\begin{array}{l}\text { Columbellidos } \\
\text { sin identificar }\end{array}$ & 2 & 1.15606936 & 1.18 & 1.7027417 & 2 & 2.27272727 & 6.49729788 & 0.85774527 \\
\hline $\begin{array}{l}\text { Portúnidos sin } \\
\text { identificar }\end{array}$ & 3 & 1.73410405 & 0.61 & 0.88023088 & 2 & 2.27272727 & 5.94167029 & 0.78439371 \\
\hline $\begin{array}{l}\text { Vitrinélido } \\
\text { sin identificar }\end{array}$ & 2 & 1.15606936 & 1.01 & 1.45743146 & 2 & 2.27272727 & 5.93977459 & 0.78414345 \\
\hline Funchalia villosa & 3 & 1.73410405 & 0.6 & 0.86580087 & 2 & 2.27272727 & 5.9088748 & 0.78006419 \\
\hline $\begin{array}{l}\text { Carídeos sin } \\
\text { identificar }\end{array}$ & 3 & 1.73410405 & 0.44 & 0.63492063 & 2 & 2.27272727 & 5.384147 & 0.71079189 \\
\hline $\begin{array}{l}\text { Isópodos } \\
\text { sin identificar }\end{array}$ & 3 & 1.73410405 & 0.25 & 0.36075036 & 2 & 2.27272727 & 4.76103274 & 0.62853103 \\
\hline Penaeus setiferus & 3 & 1.73410405 & 1.09 & 1.57287157 & 1 & 1.13636364 & 3.75792684 & 0.49610531 \\
\hline $\begin{array}{l}\text { Anchoviella } \\
\text { perfasciata }\end{array}$ & 1 & 0.57803468 & 1.51 & 2.17893218 & 1 & 1.13636364 & 3.13291689 & 0.41359419 \\
\hline $\begin{array}{l}\text { Decápodos } \\
\text { sin identificar }\end{array}$ & 2 & 1.15606936 & 0.08 & 0.11544012 & 2 & 2.27272727 & 2.88979427 & 0.38149819 \\
\hline $\begin{array}{l}\text { Penaeus } \\
\text { duorarum }\end{array}$ & 1 & 0.57803468 & 0.61 & 0.88023088 & 1 & 1.13636364 & 1.65711996 & 0.21876584 \\
\hline Callinectes sp. & 1 & 0.57803468 & 0.48 & 0.69264069 & 1 & 1.13636364 & 1.44394929 & 0.19062396 \\
\hline $\begin{array}{l}\text { Corbúlidos } \\
\text { sin identificar }\end{array}$ & 2 & 1.15606936 & 0.03 & 0.04329004 & 1 & 1.13636364 & 1.36290842 & 0.1799253 \\
\hline $\begin{array}{l}\text { Ofídidos } \\
\text { sin identificar }\end{array}$ & 1 & 0.57803468 & 0.4 & 0.57720058 & 1 & 1.13636364 & 1.31276734 & 0.17330589 \\
\hline
\end{tabular}




\begin{tabular}{|c|c|c|c|c|c|c|c|c|}
\hline Entidad & $\mathbf{N}$ & N \% & $\begin{array}{c}\text { Peso } \\
\text { (g) }\end{array}$ & P \% & FO & FO \% & IIR & IIR \% \\
\hline $\begin{array}{l}\text { Hepatus } \\
\text { epheliticus }\end{array}$ & 1 & 0.57803468 & 0.39 & 0.56277056 & 1 & 1.13636364 & 1.2963696 & 0.17114113 \\
\hline Sicyonia sp. & 1 & 0.57803468 & 0.16 & 0.23088023 & 1 & 1.13636364 & 0.91922149 & 0.12135166 \\
\hline Hepatus sp & 1 & 0.57803468 & 0.14 & 0.2020202 & 1 & 1.13636364 & 0.886426 & 0.11702214 \\
\hline Upogebia sp. & 1 & 0.57803468 & 0.06 & 0.08658009 & 1 & 1.13636364 & 0.75524406 & 0.09970407 \\
\hline $\begin{array}{l}\text { Olívidos } \\
\text { sin identificar }\end{array}$ & 1 & 0.57803468 & 0.01 & 0.01443001 & 1 & 1.13636364 & 0.67325534 & 0.08888027 \\
\hline $\begin{array}{l}\text { Anomuros } \\
\text { sin identificar }\end{array}$ & 1 & 0.57803468 & 0 & 0 & 1 & 1.13636364 & 0.65685759 & 0.08671551 \\
\hline $\begin{array}{l}\text { Bivalvos } \\
\text { sin identificar }\end{array}$ & 1 & 0.57803468 & 0 & 0 & 1 & 1.13636364 & 0.65685759 & 0.08671551 \\
\hline $\begin{array}{l}\text { Nasáridos } \\
\text { sin identificar }\end{array}$ & 1 & 0.57803468 & 0 & 0 & 1 & 1.13636364 & 0.65685759 & 0.08671551 \\
\hline Total & 173 & 100 & 69.3 & 100 & 88 & 100 & 757.485712 & 100 \\
\hline
\end{tabular}

En el primer período de muestreo se encontró un total de 19 tipos alimentarios, solo uno perteneció al grupo de los peces (Osteichthyes: Ophididae) y otro al de los moluscos (Gasteropoda; Olivellidae), el resto fueron crustáceos. En contraste a la colecta del segundo período de muestreo (octubre), donde se encontraron al menos tres especies de peces, cinco taxa de moluscos, y el resto fueron de crustáceos, sumando un total de 22 tipos alimentarios y 18 distintos a los del primer muestreo (Fig.1).

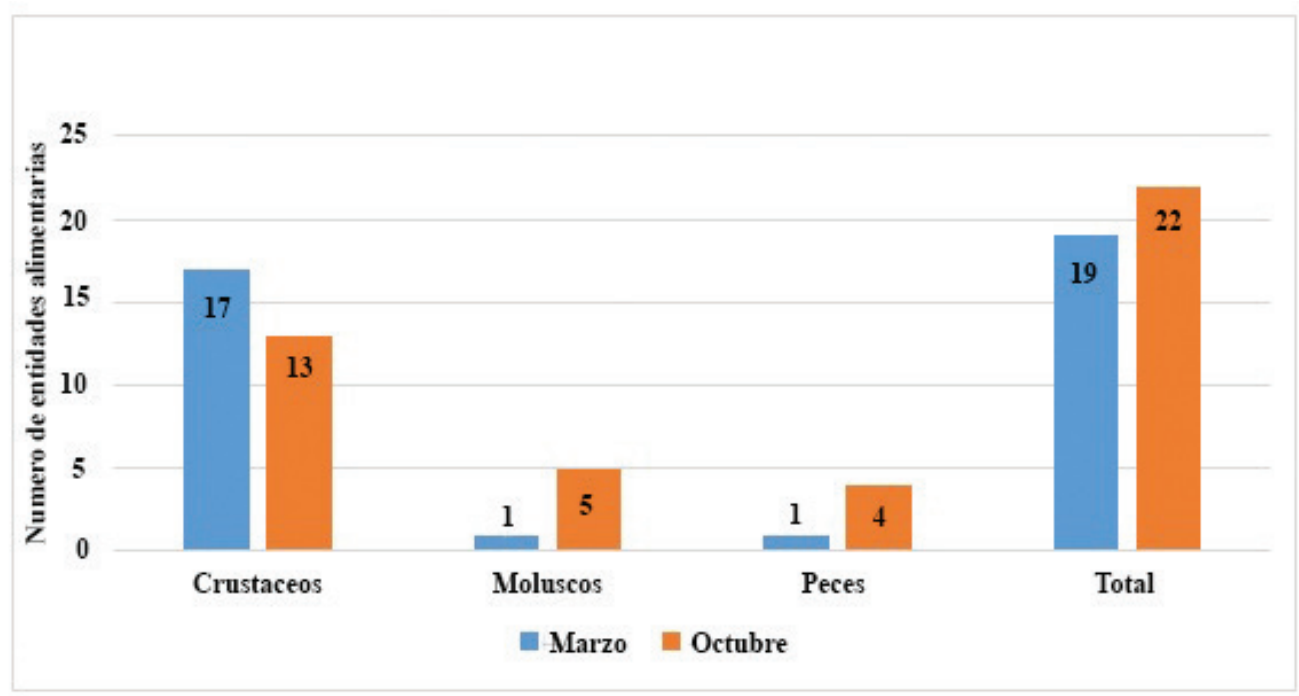

Figura 1. Número de tipos alimentarios de los grupos que comprendieron la dieta de Prionotus rubio en los períodos de muestreo. 
Para hacer más clara la información presentada sobre la importancia relativa de los grupos alimentarios, estos fueron dispuestos en diversas categorías para su análisis y representación gráfica. En la Figura 2, se muestra el IIR por categoría, en el cual se observa que el grupo de los crustáceos (camarones, camarones mantis y jaibas) corresponden a casi el $70 \%$ de los tipos alimentarios de $P$. rubio.

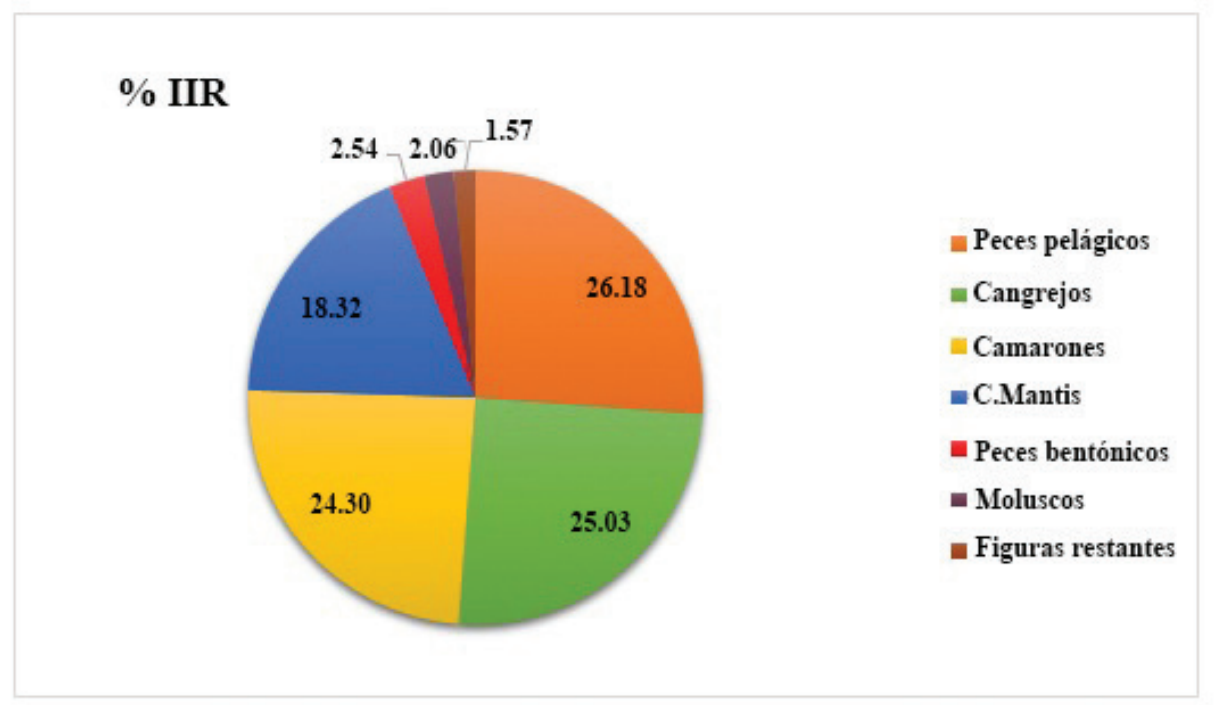

Figura 2. Grupos de los tipos alimentarios más importantes en porcentaje de IIR en todo el periodo de muestreo.

En la colecta del mes de marzo, los tipos alimentarios con mayor IIR fueron los cangrejos, seguido de camarones y por último los cangrejos mantis (Fig. 3). En octubre, el más destacado fue el de peces pelágicos, seguido de cangrejos, camarones, peces bentónicos y por último moluscos (Fig. 4).

Las especies con mayor importancia (\% IIR), fueron distintas entre ambas investigaciones; en el estudio del 2001, Peaneus aztecus comprendió el 42 \%, Sycionia dorsalis el $21 \%$ y Portunus spinicarpus el $20 \%$, entre los decápodos; en cambio, en el presente estudio los tipos alimentarios, a nivel de especie con mayor importancia, fueron Anchoa sp. $20 \%$, Sicyonia typica $8 \%$ y Squilla sp. 7 \% (Tabla I). Las diferencias podrían deberse a las condiciones ambientales del año en los que se efectuaron los estudios, puesto que hay más de 20 años de diferencia entre los muestreos de ambos estudios; además la técnica de muestreo también pudo afectar ya que en aquel estudio realizaron el arrastre mediante una lancha y en mar abierto, y en el presente fue en la playa y a muy baja profundidad.

Autores como Schmitter-Soto y Castro-Aguirre (1996), hicieron alusión a que la variación de las dietas, entre especies, puede fluctuar por su distribución batimétrica y latitudinal; podemos ver esta variación incluso para la misma especie en ambientes distintos. 


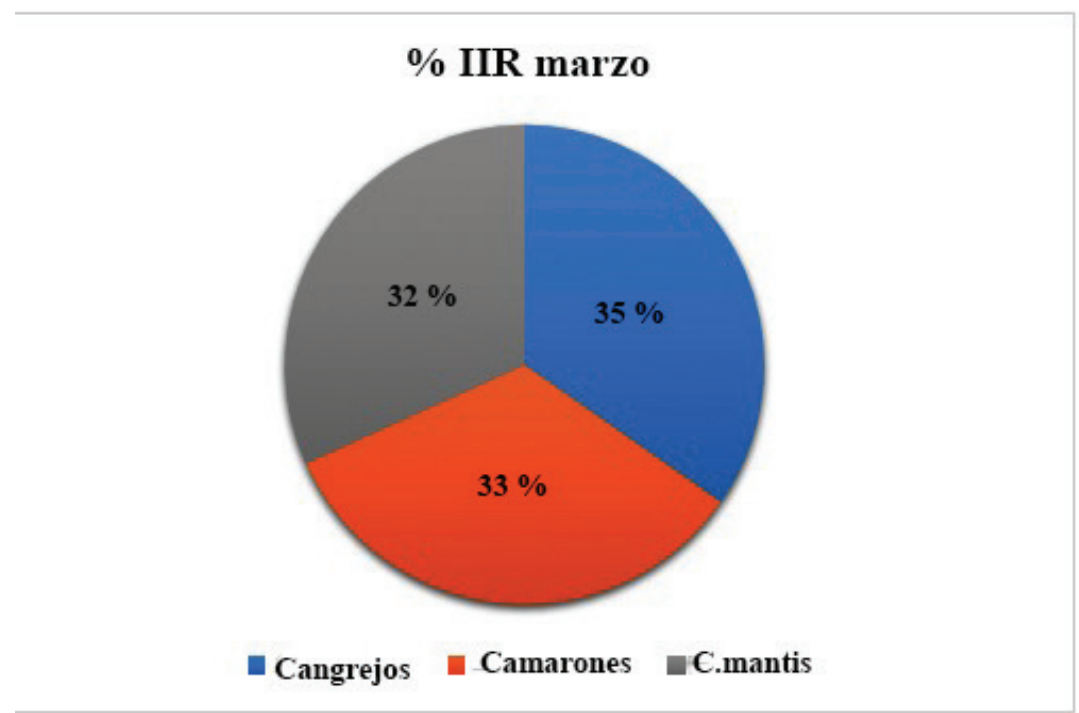

Figura 3. Grupos de los tipos alimentarios más representativas del mes de marzo en porcentaje de IIR.

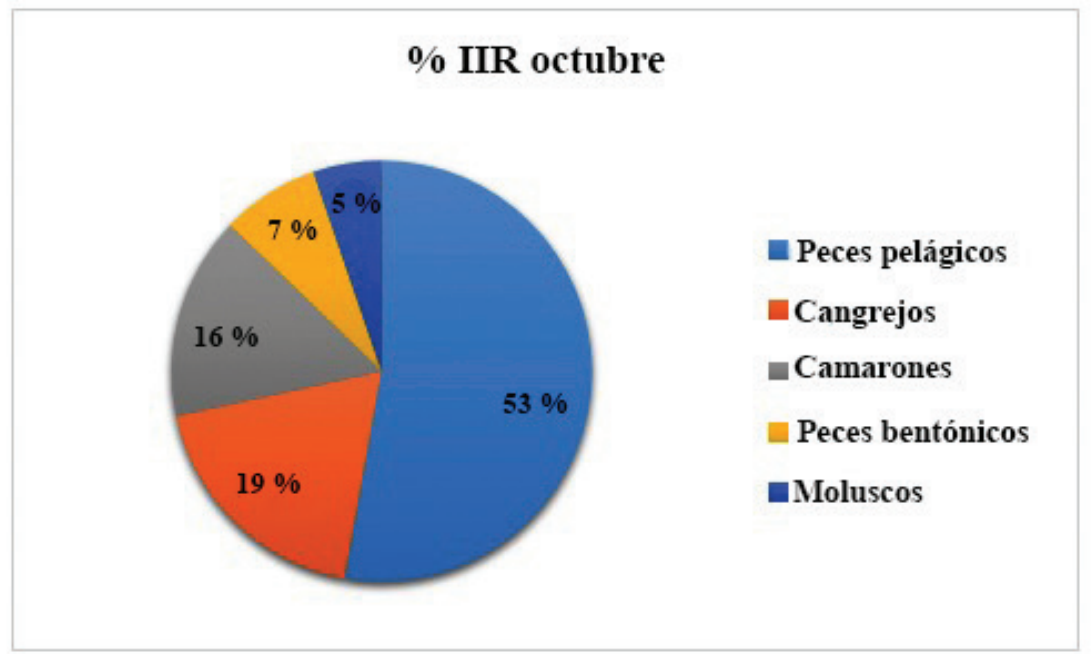

Figura 4. Entidades alimentarias más representativas del mes de octubre en porcentaje de IIR.

El índice de Levins para el mes de marzo fue de 0.67 , y de 0.77 para el mes de octubre, con un total general de 0.69 , un valor considerado propio de organismos generalistas, lo cual sugiere que no tiene preferencia por algún tipo alimentario. En cuanto al nivel trófico, se obtuvo un resultado de 3.9, por lo que está situado como un carnívoro de nivel medio, según Froese y Pauly (2019; Fig. 6). 


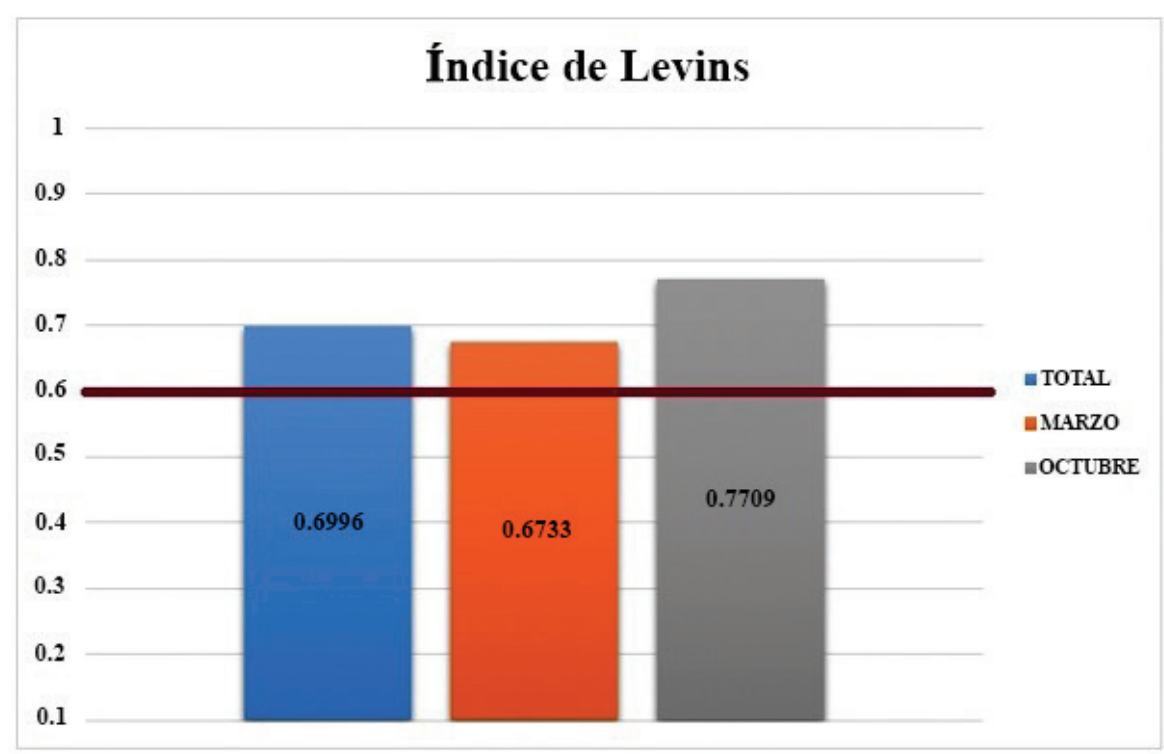

Figura 5. Índice Levins para Prionotus rubio, en los distintos periodos de muestreo. La línea roja representa la cifra a partir de la cual se considera al organismo como generalista.

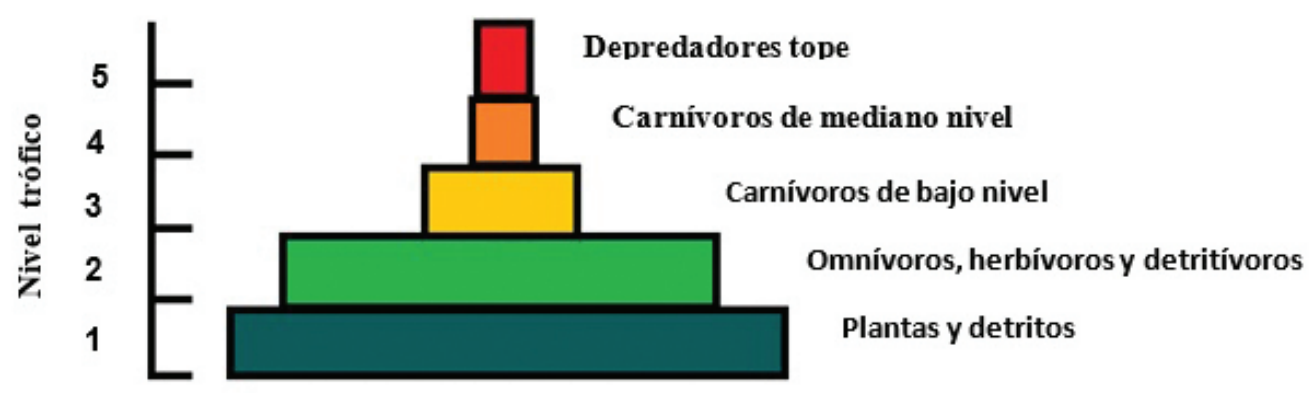

Figura 6. Muestra los niveles tróficos y su denominación según la FAO, en la región del Atlántico Oeste Central, para México. Tomado de Fishbase.org.

A nivel cualitativo, se puede observar que la especie en estudio se alimenta principalmente de fauna bentónica, no obstante, es imprescindible contar con el respaldo estadístico o numérico que confirme lo antes mencionado. Por eso, para interpretar mejor los tipos alimentarios de esta especie, los índices son una herramienta muy útil. De esta manera, se concluye que esta especie, en la localidad de estudio, no tiene preferencia hacia algún tipo alimentario, pero la mayoría de sus presas son crustáceos. Debido a los resultados estimados con los índices, se considera como una especie carnívora generalista y los tipos alimentarios en su dieta dependen de la abundancia de las presas en las diferentes temporadas. 


\section{AGRADECIMIENTOS}

A los pescadores de Las Barrancas por apoyarnos con la captura de los peces. A Ricardo Olmos por apoyarnos con la identificación de los moluscos. A Carlos Blas Garfias y otros compañeros de la Facultad de Estudios Superiores Iztacala, que apoyaron con comentarios y recomendaciones para este trabajo. A los revisores anónimos de Novitates Caribaea.

\section{LITERATURA CITADA}

Abbott, R. T. 1974. American Seashells; The marine Molluska of the Atlantic and Pacific Coasts of North America. Van Nostrand Reinhold Company, New York, USA. ISBN: 978-0442202286; 663 pp.

Abele, L. G. y W. Kim. 1986. An illustrated guide to the marine decapod crustaceans of Florida (Vol. 8). State of Florida, Department of Environmental Regulation, USA. 1-209 pp.

Cortés, E. 1999. Standardized diet compositions and trophic levels of sharks. ICES. Journal of Marine Science, 56 (5): 707-717. doi.org/10.1006/jmsc.1999.0489.

Cruz-Escalona, V. H., L. A. Abítia-Cárdenas, L. Campos-Dávila y F. Galvan-Magaña. 2001. Feeding Ecology of the Blackwing Searobin Prionotus rubio (Jordan, 1886) Over the Western Continental Shelf off Alvarado, Veracruz, Mexico. Gulf of Mexico Science, 19 (1): 1. doi: .org/10.18785/goms.1901.01.

Froese, R. y D. Pauly (Eds.). 2019. FishBase. World Wide Web electronic publication. Recuperado el 18 de febrero del 2018, en https:/www.fishbase.se/TrophicEco/trophicanalysis. php? requesttimeout $=18000 \& \mathrm{vc}$ _code $=484 \&$ vareacode $=31 \&$ tag $=2 \& \mathrm{ht}=\& \mathrm{ft}=$.

Gore, R. H y L. E. Scotto. 1979. Crabs of the family Parthenopidae (Crustacea Brachyura: Oxyrhyncha) with notes on specimens from the Indian River region of Florida. En: Memoirs of the Hourglass Cruises. Vol III, Part VI. 98 pp.

Hoese, H. D. y R. H. Moore. 1977. Fishes of the Gulf of Mexico, Texas, Louisiana and adjacent waters. Texas A \& M Univ. Press, College station, TX. 287-291 pp.

Hyslop, E. J. 1980. Stomach contents analysis-a review of methods and their application. Journal of Fish Biology, 17: 411-429. doi: .org/10.1111/j.1095-8649.1980.tb02775.x.

Krebs, C. J. 1999. Ecological methodology. Addison Wesley Longman. USA, 620 pp.

Lewis, C. T. y W. R. Yerger. 1976. Biology of five species of searobin (Pisces: Triglidae) from the northeastern Gulf of Mexico. Fish Bulletin, 4 (1): 93-103.

Manning, R. B. 1969. Stomatopoda crustacea of the Western Atlantic. University of Miami Press, USA. 380 pp. doi: https://doi.org/10.2307/1295219.

McEachran, J. D. y J. D. Fechhelm. 2005. Fishes of the Gulf of Mexico. Vol. 2. Austin: University of Texas Press, USA. 1004 pp.

Miller, G. C. y W. J. Richards. 1977. Triglidae. En: Fischer, W. (1978). FAO species identification sheets for fishery purposes. Western Central Atlantic (fishing area 31). No. F010. 024. 
Ng, P. K., D. Guinot y P. J. Davie. 2008. Systema Brachyurorum. Part 1. An annotated check list of extant Brachyuran crabs of the word. The Raffles Bulletin of Zoology, 17: 1-286.

Ortiz, M. y R. Lalana. 2010. Claves taxonómicas para identificar a crustáceos cubanos (Arthropoda, Crustacea). Cocuyo, 18: 5-28.

Peláez-Rodríguez, E., J. Franco-López, W. A. Matamoros, R. Chavez-López y N. J. Brown-Peterson. 2005. Trophic relationships of demersal fishes in the shrimping zone off Alvarado Lagoon, Veracruz, Mexico. Gulf and Caribbean Research, 17 (1): 157-167 doi. org/10.18785/gcr.1701.16.

Pinkas, L., M. S. Oliphant y I. L. K. Iverson. 1971. Food habits study. Food habits of albacore bluefin tuna and bonito in California waters. Fish Bulletin, 152: 1-105.

Randall, J. E. 1983. Caribbean Reef Fishes. T. F. H Publ. Inc. 350. doi: https://doi. org/10.2307/1441728.

Ramírez-Rodríguez, M. 2015. La pesquería de camarón en Campeche: Desarrollo histórico y perspectiva. Ciencia Pesquera, 23 (1): 73-87.

Ross, J. 1983. Memoirs of the hourglass cruises. Searobins (Triglidae). Marine Research Laboratory, 6: 1-76.

Richards W.J. y G. C. Miller. 2002. Triglidae. In E. K, Carpenter(ed). The living marine resources of the Western Central Atlantic. Vol. 2. Bony fishes part 1 (Acipenseridae to Grammatidae). FAO Species Identification Guide for Fishery Purposes an American Society of Ichthyologists and Herpetologists Special Publication No. 5. Rome, FAO. 1266-1277 pp.

Silver, W. L. y T. E. Finger. 1984. Electrophysiological examination of a non-olfactory, non-gustatory chemosense in the searobin Prionotus carolinus. J. Comp. Physiol. A: Neuroethology, Sensory, Neural, and Behavioral Physiology, 154 (2), 167-174. doi: https://doi.org/10.1007/bf00604982.

Schmitter-Soto, J. J. y J. L. Castro-Aguirre. 1996. Trophic comparison among Triglidae (Pisces: Scorpaeniformes) off Baja California Sur, Mexico. Revista de Biología Tropical, 44 (2): 803-811.

Tunnell Jr, J.W., J.Andrews, N. C. Barrera y F. Moretzsohn. 2010. Encyclopedia of Texas seashells, identification, ecology, distribution \& history. Texas A\&M University Press.104-276 doi: https://doi.org/10.5860/choice.48-2428.

[Recibido: 28 de mayo, 2019. Aceptado para publicación: 23 de octubre, 2019] 